\title{
A geomagnetic total intensity anomaly originated from lightning-induced isothermal remanent magnetization: case of the Yatsugatake Magnetic Observatory, central Japan
}

\author{
Hisayoshi Shimizu, Takao Koyama, Shigeru Koyama, and Hisashi Utada \\ Earthquake Research Institute, University of Tokyo, Tokyo, Japan
}

(Received July 7, 2006; Revised October 16, 2006; Accepted October 26, 2006; Online published March 23, 2007)

\begin{abstract}
Magnetic total intensity anomaly maps of the Yatsugatake Magnetic Observatory, central Japan, in 1975, 1982 and 2005 are examined. The magnetic environment seems to be stable during this period except for the appearance of an anomalous thin patch-pair in the middle part of the observatory sometime between 1975 and 1982. The peak-to-peak amplitude of the patch-pair is about $80 \mathrm{nT}$ at $2 \mathrm{~m}$ above ground level. The magnetization induced by the horizontal electric current in the ground due to the lightning which hit the observatory area in July 1981 is a probable cause for the patch-pair. Modeling confirmed that the characteristics of the patch-pair are well reproduced by model magnetizations that represent lightning-induced isothermal remanent magnetization acquired from horizontal lightning electric current in the ground. The required maximum magnetization to explain the peak-to-peak amplitude of the patch-pair is estimated to be in the range of 10 to $40 \mathrm{~A} / \mathrm{m}$.
\end{abstract}

Key words: Magnetic anomaly map, lightning-induced isothermal remanent magnetization, magnetic environment, total intensity.

\section{Introduction}

The Yatsugatake Magnetic Observatory (YMO; $36^{\circ} 04^{\prime} \mathrm{N}$, $138^{\circ} 27^{\prime} \mathrm{E}$, altitude: $1170 \mathrm{~m}$ a.s.l.) has been in operation since 1970 as a reference geomagnetic station in the central part of Japan (see Fig. 1), and the magnetic field data collected there have been utilized for monitoring crustal activities in the Izu and Tokai regions and for studies of volcano magnetism, electrical conductivity structures, among others.

The magnetic environment around such reference observatories should be calm and stable: the spatial gradient of the magnetic field around working magnetometers should be small, and the magnetic field recorded should not change drastically over time. The total magnetic field anomaly map, which presents a spatial distribution of the total magnetic intensity relative to a reference, is often used to monitor the magnetic environment. In this paper, we present a newly obtained magnetic anomaly map for YMO in 2005 together with those obtained in 1975 and 1982. Based on these maps, the magnetic environment can be seen to have been suitable and stable at the locations where sensors for continuous magnetic measurements were installed. However, there was a significant change in magnetic anomaly, which attracted our attention, in a part of the YMO.

As is shown in Section 2, a thin anomalous patch-pair extending east-west occurs in the middle part of the site in the 1982 and 2005 maps which is not found in the 1975 map. A probable cause of this patch-pair is magnetization induced

Copyright (c) The Society of Geomagnetism and Earth, Planetary and Space Sciences (SGEPSS); The Seismological Society of Japan; The Volcanological Society of Japan; The Geodetic Society of Japan; The Japanese Society for Planetary Sciences; TERRAPUB by a lightning electric current (lightning-induced isothermal remanent magnetization, LIRM; see Cox (1961)) that flowed in the ground. Indeed, there was a severe lightning strike in the observatory area on July 16, 1981 that caused severe damage to the instruments in the observatory and step-like changes in the intensity of the magnetic field over the YMO area (Utada and Koyama, 1982). The immediate effect and short-term after-effect of the lightning strike are summarized in Section 3.

Although a number of studies have detected LIRMinduced magnetic field anomaly (e.g. Jones and Maki, 2005), these have not shown if the LIRM of a possible intensity and proper direction can generate an observed distribution of magnetic anomalies. The objective of this paper is to examine as quantitatively as possible whether the anomalous patch-pair in YMO can be regenerated by model distributions of LIRM near the surface acquired by plausible lightning electric current.

\section{Magnetic Anomaly Maps}

The main observation site of the YMO is a $60 \times 55-\mathrm{m}$ rectangle region that is bounded by a $100 \times 100-\mathrm{m}$ region to keep sensors away from man-made disturbances. The inner rectangular region is the site of the present study. A variometer house $(\mathrm{V})$ in which two sets of fluxgate magnetometers are installed, an absolute measurement house (Ab) and a recording house $(\mathrm{R})$ are located in the site, as shown in Fig. 2. Sensor locations for present and past proton magnetometers are also designated in Fig. 2 as A, B, C, D, 1, 2 and P1. The surface layer of the area is a thick loam layer composed of Pleistocene volcanic ash from the Yatsugatake volcanoes (Aida, 1978). The loam layer is more than $5 \mathrm{~m}$ 
Yatsugatake 2005

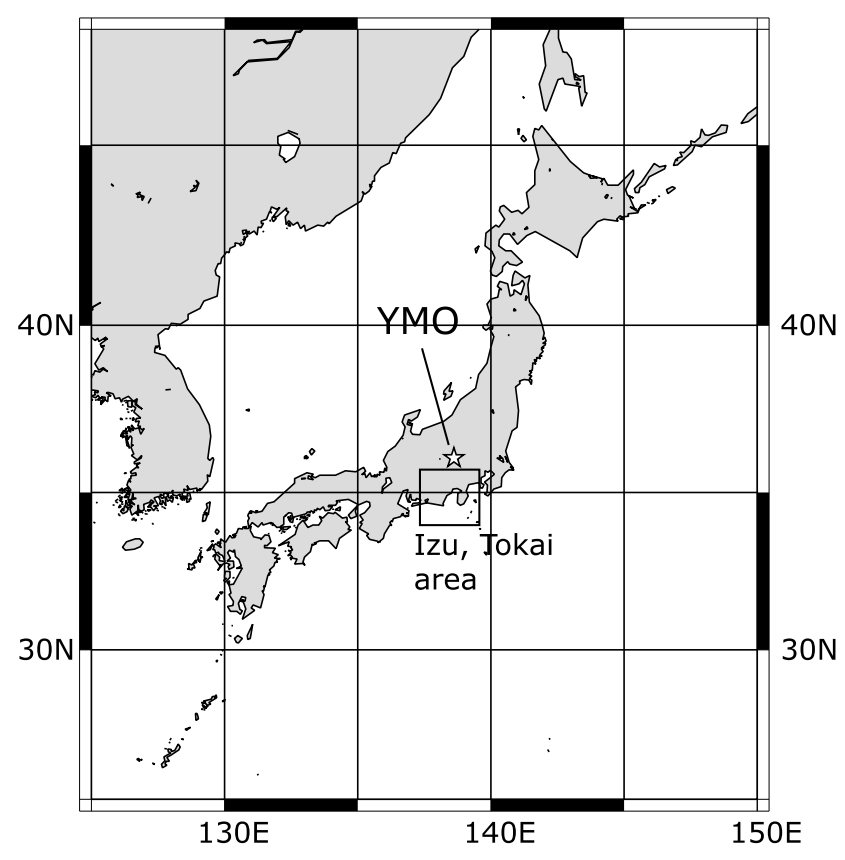

Fig. 1. Location of the Yatsugatake Magnetic Observatory (YMO) and $\mathrm{Izu} /$ Tokai area in central Japan.

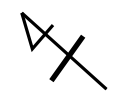

${ }^{\mathrm{B}}$
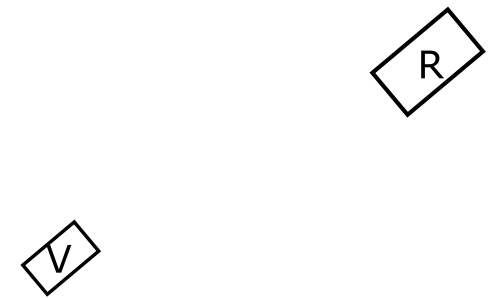

A
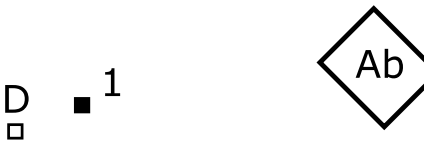

C

口

P1

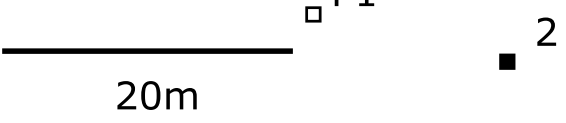

Fig. 2. Layout of facilities in the YMO. $\mathrm{R}$ is the recording house, $\mathrm{V}$ is the variameter house where fluxgate sensors are installed, $\mathrm{Ab}$ is the absolute measurement house, A, B, C, D, P1, 1 and 2 are locations for permanent and temporal sites for proton magnetometers.

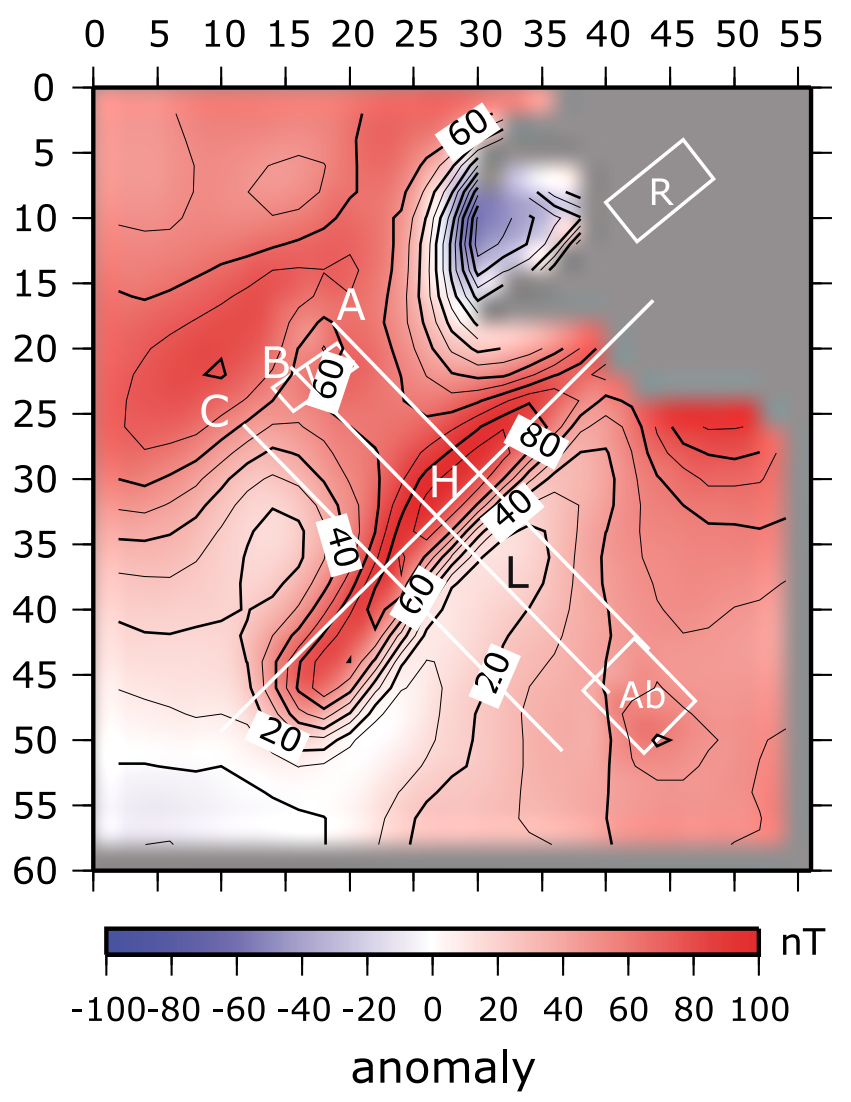

Fig. 3. Magnetic anomaly map in YMO for 2005. Reference is P1 (see Fig. 2). Unit: nT.

thick where the variometer house $(\mathrm{V})$ has a vault for fluxgate sensors. The natural remanent magnetization of the surface layer in the eastern bank of Yatsugatake, where the observatory is located, is in the range of $0.1-1 \mathrm{~A} / \mathrm{m}$ (Yukutake et al., 1975; Aida, 1978). Other magnetic properties have not been reported in the literature.

A recent survey of magnetic total intensity in the site was carried out during December 13-15, 2005 using a portable Overhauser-type proton precession magnetometer. In this survey, measurements of the geomagnetic intensity were made at $2 \times 2-\mathrm{m}$ grid points in the observatory area. The sensor height was kept at $2 \mathrm{~m}$ above the ground. Observed intensities were compared to the total intensity simultaneously measured at P1 in order to obtain a magnetic anomaly map. The magnetic field anomaly is defined as

$$
\begin{aligned}
\Delta F(x, y) & \approx \Delta F(x, y ; t) \\
& =F_{\text {Grid }}(x, y ; t)-F(P 1 ; t) .
\end{aligned}
$$

The effect of external field variations is almost cancelled by taking the difference. The grid points were determined carefully, but there may be a location error of up to several tens of centimeters in the observation.

The magnetic anomaly map in the site for 2005 is presented in Fig. 3. The magnetic field in the region close to the recording house $\mathrm{R}$ is disturbed significantly by the house itself and electromagnetic noise originating from the power line. The areas around $\mathrm{V}, \mathrm{Ab}, \mathrm{B}$ and $\mathrm{C}$ have a rel- 


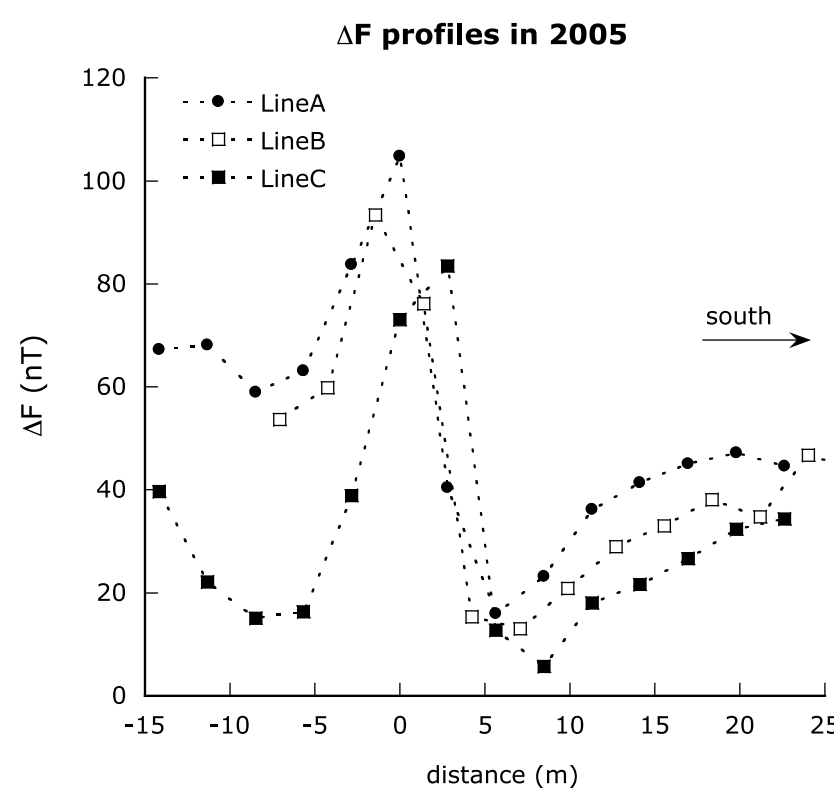

Fig. 4. Profiles of magnetic field anomaly along lines $\mathrm{A}, \mathrm{B}$, and $\mathrm{C}$ in Fig. 3.

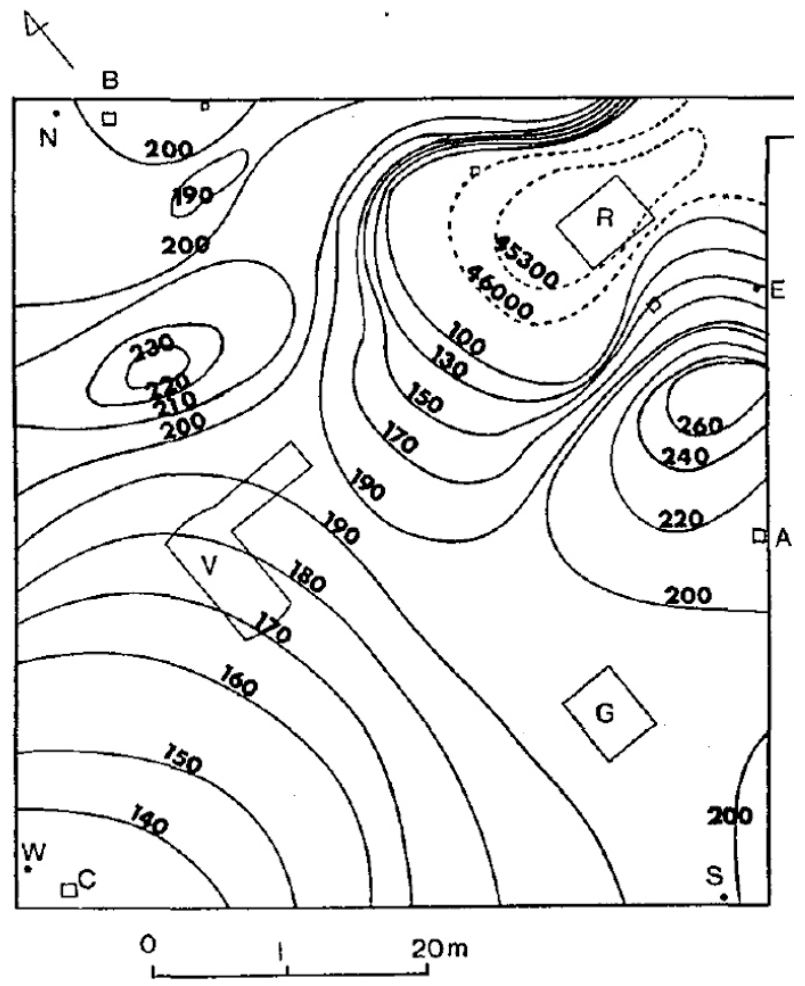

Fig. 5. Magnetic field intensity map in YMO for 1975 (from Yukutake et al., 1975). Unit: nT.

atively small magnetic gradient, about $1-2 \mathrm{nT} / \mathrm{m}$, and it may be concluded that these places are suitable locations for magnetic sensors. Conversely, there is a strong anomalous patch-pair spreading in the east-west direction in the anomaly map ( $\mathrm{H}$ and $\mathrm{L}$ in Fig. 3). Figure 4 shows the profiles of magnetic anomaly across the patch-pair along lines $\mathrm{A}, \mathrm{B}$, and $\mathrm{C}$ in Fig. 3. The peak-to-peak difference in the
Yatsugatake 1982

$\begin{array}{llllllllllll}0 & 5 & 10 & 15 & 20 & 25 & 30 & 35 & 40 & 45 & 50 & 55\end{array}$

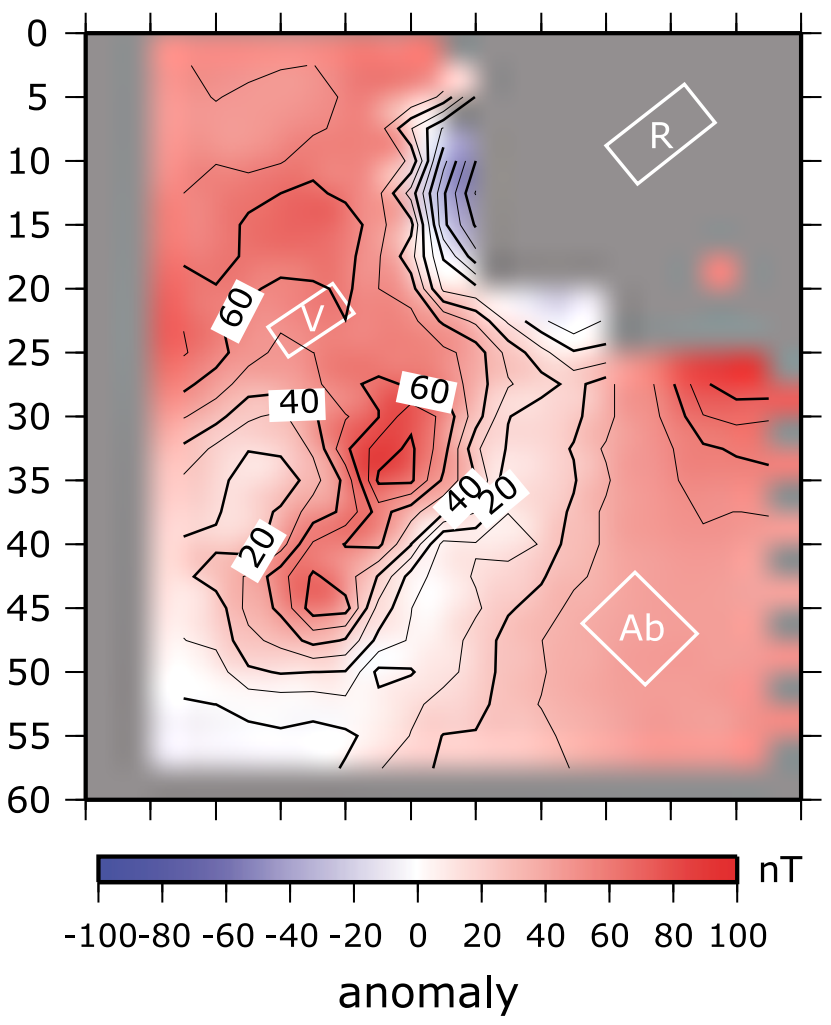

Fig. 6. Magnetic anomaly map in YMO for 1983 (unpublished data). Reference is P1. Unit: nT.

intensity is about $80 \mathrm{nT}$, and the separation of the peaks is about $5 \mathrm{~m}$. The resultant magnetic field gradient is about $15 \mathrm{nT} / \mathrm{m}$ at its maximum. The patch-pair is confined to a narrow zone of $15 \mathrm{~m}$ in width in the north-south direction.

The 1975 magnetic field total intensity map (Yukutake et al., 1975) and the 1982 magnetic anomaly map in terms of P1 (unpublished data) are shown in Figs. 5 and 6. The grid spacing for the survey is $5 \mathrm{~m}$ for both directions, and the height of the observation is $1.5 \mathrm{~m}$. It is evident that the anomalous patch-pair in the middle of the observatory area does not exist in the map for 1975, but it does appear in that for 1982. The overall features of the magnetic anomaly maps seem to be similar to each other for all three maps if the patch-pair is removed from the 1982 and 2005 maps.

The maps and anomalies for 1982 and 2005 can be examined more closely and quantitatively. The difference in the magnetic anomaly maps between 2005 and 1982 is shown in Fig. 7, in which $2 \times 2$-m grid data for 2005 were down-sampled at the $5 \times 5$-m grid for comparison. The average of the differences at the grid points is $3.7 \mathrm{nT}$. The locations having relatively large differences are situated at places where both the magnetic anomaly and its spatial gradient are large. Although it is possible that some of the patches in Fig. 7 are due to slight changes in magnetization, it seems natural to attribute the causes to the height difference of the observations with the magnetic source at very shallow depth and/or the location errors during magnetic 
Table 1. Size of steps in total intensity in comparison to that observed at the Kakioka Magnetic Observatory (reproduction of Table 1 by Utada and Koyama 1982).

\begin{tabular}{c|c|c|c}
\multirow{2}{*}{ Site } & \multirow{2}{*}{ Size of step (nT) } & Before the lightning & Observation period \\
\cline { 2 - 4 } B & +0.5 & June 1-July 15, 1981 & October 17-22, 1981 \\
$\mathrm{C}$ & +2.3 & June 1-July 15, 1981 & October 17-22, 1981 \\
$\mathrm{D}$ & -5.9 & June 1-July 15, 1981 & September 18, 1981 \\
1 & -22.4 & June 1-July 15, 1981 & August 20-September 30, 1981 \\
2 & -3.4 & June 1-July 15, 1981 & August 1-September 30, 1981 \\
A & +1.6 & June 1-July 15, 1981 & October 17-22, 1981
\end{tabular}

\section{5-1982}

\section{$\begin{array}{llllllllllll}0 & 5 & 10 & 15 & 20 & 25 & 30 & 35 & 40 & 45 & 50 & 55\end{array}$}

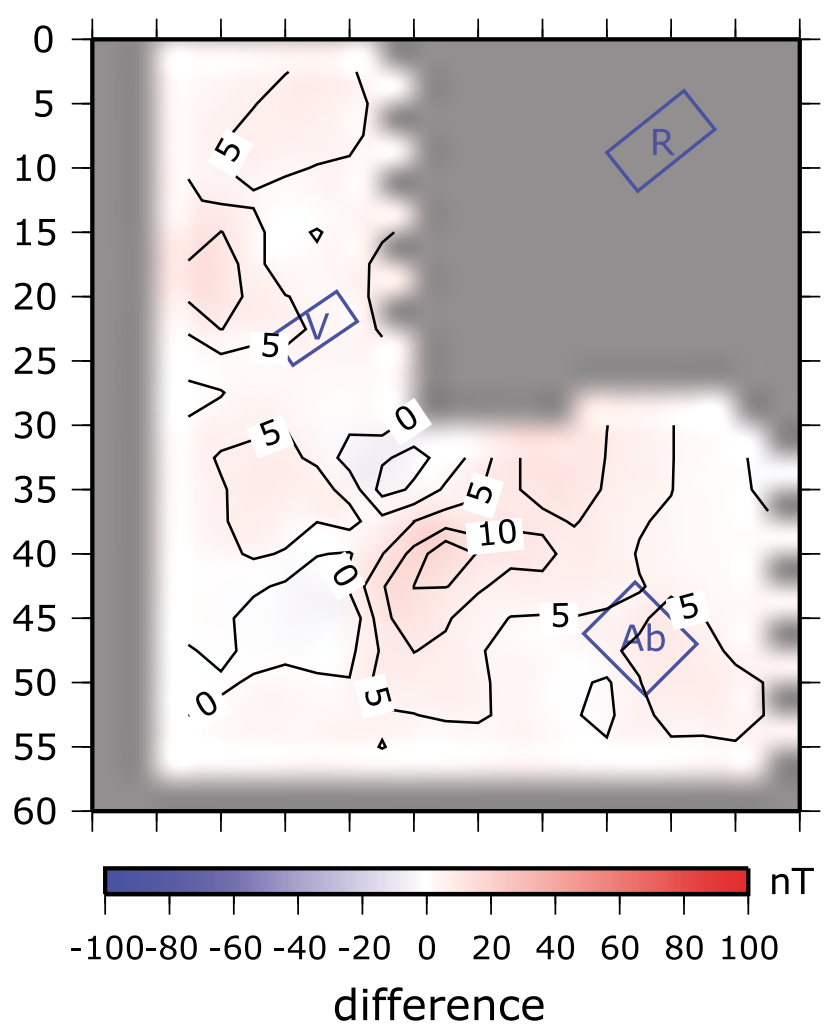

Fig. 7. Difference of magnetic field anomaly map for 2005 and 1982. Unit: nT.

field mapping with the effect of down-sampling during the process of calculating the differences. We will reexamine the cause of the difference in Section 5.

\section{Probable Cause of the Patch-Pair: LIRM}

The anomalous patch-pair that is evident in the 1982 and 2005 maps indicates that a shallower part of the ground in the middle of the site was locally magnetized sometime between 1975 and 1982. In general, tectonically induced and/or artificially placed magnetization are potential causal candidates of magnetization; however, in the present case, the patch-pair is so confined that it would be impossible to generate by tectonic activities. Also, the site is well main- tained so that there is nothing to cause extra magnetization artificially. As such, the only reasonable cause of the magnetization generating the patch-pair seems to be LIRM acquired by the lightning electric current due to the severe lightning strike in the site on July 16, 1981.

Details of the direct and short-term after-effects of the lightning are summarized in Utada and Koyama (1982). According to these authors, a lightning strike occurred somewhere in the observatory area on July 16, 1981, but it was not possible to identify the exact place where the lightning hit. The lightning strike did cause severe damage to the electric circuits and power supply systems of the observatory, with all of the instruments operative at the moment of the lightning strike rendered inoperative due to the damage. Observations of total magnetic intensity at sites 1 and 2 using portable proton magnetometers were restarted after a few days, but observations at sites $\mathrm{A}, \mathrm{B}$ and $\mathrm{C}$ were not resumed for 3 months because of the severe damage inflicted on these proton magnetometers. The steps of the total intensity before and after the lightning strike were clearly visible in the time series of the observed magnetic field. For example, the difference in the magnetic intensity at site 1 and 2 (see Fig. 2) in reference to that at the Kakioka Magnetic Observatory changed by $-22.4 \mathrm{nT}$ and $-3.4 \mathrm{nT}$, respectively, before and after the lightning. The size of the steps at other sites is summarized in Table 1 (reproduction of Table 1 by Utada and Koyama (1982)). It is also evident that the difference in the total intensity between sites 1 and 2 decreased by about $1 \mathrm{nT}$ in the following 2 months after the lightning strike but that this became somewhat constant thereafter (Fig. 8).

The observations by Utada and Koyama (1982) seem to indicate that some part of the loam soil of the ground, probably somewhere close to site 1 , received LIRM. In addition, the time series shown in Fig. 8 suggests that a part of the magnetization was unstable and re-magnetized by viscous remanent magnetization. However, the amount of the change in the magnetic intensity between site 1 and site 2 due to the effect of viscous remanent magnetization (about $1 \mathrm{nT}$ ) is very small compared with the step before and right after the lightning strike (about $20 \mathrm{nT}$ ), which indicates that the LIRM in the site is stable. A stable LIRM provides further support for the similarity of the magnetic anomaly map for 1982 and 2005 if the patch-pair is generated by the LIRM. 


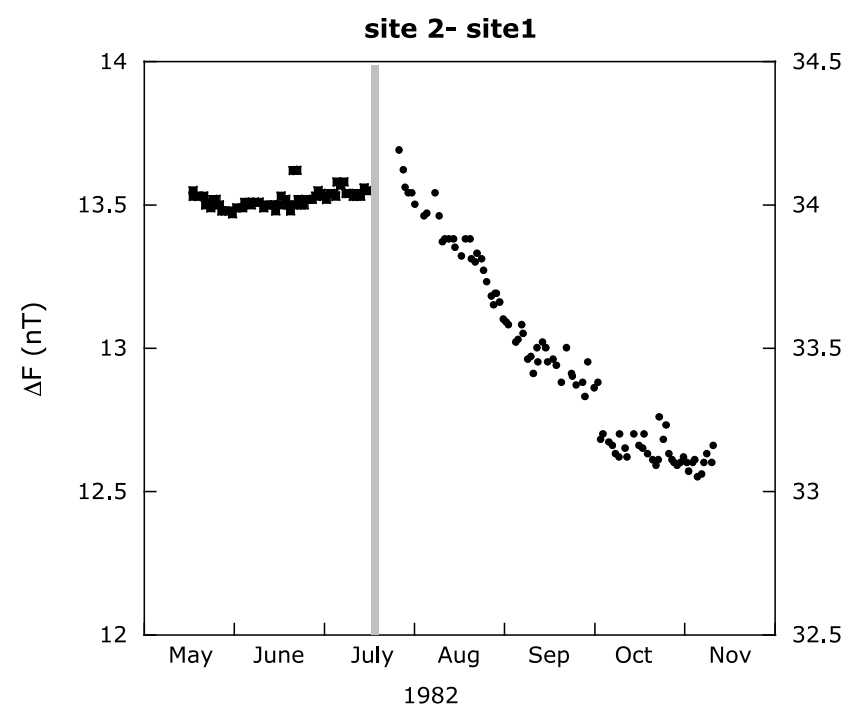

Fig. 8. Difference in the total intensity at site 2 and 1 (see Fig. 2) before and after the lightning (reproduction of Fig. 3 of Utada and Koyama, 1982). The label on the left is for the difference before July 16th, and that on the right is for those after the lightning. There is a step of about $20 \mathrm{nT}$ immediately before and after the lightning.

\section{Modeling of Magnetization and Magnetic Anomaly}

Let us examine if the anomalous patch-pair can be generated by a physically plausible distribution of LIRM. We are going to calculate magnetic flux density $\mathbf{B}$ by specifying magnetization $\mathbf{J}(\mathrm{A} / \mathrm{m})$ and using the following relationship

$$
\mathbf{B}(\mathbf{x})=-\frac{\mu_{0}}{4 \pi} \int_{V^{\prime}}\left(\mathbf{J}\left(\mathbf{x}^{\prime}\right) \cdot \nabla\right) \frac{\left(\mathbf{x}-\mathbf{x}^{\prime}\right)}{\left|\mathbf{x}-\mathbf{x}^{\prime}\right|^{3}} d^{3} \mathbf{x}^{\prime}
$$

which is derived from

$$
\mathbf{B}(\mathbf{x})=-\nabla \psi(\mathbf{x}), \quad \psi(\mathbf{x})=-\frac{\mu_{0}}{4 \pi} \int_{V^{\prime}} \frac{\nabla_{\mathbf{x}^{\prime}} \cdot \mathbf{J}\left(\mathbf{x}^{\prime}\right)}{\left|\mathbf{x}-\mathbf{x}^{\prime}\right|} d^{3} \mathbf{x}^{\prime}
$$

where $\mu_{0}$ is the magnetic permeability of the vacuum, $\psi$ is a scalar magnetic potential, and $V^{\prime}$ is the volume occupied by magnetization.

LIRM is an isothermal magnetization obtained due to a very strong magnetic field accompanied by lightning electric current in the ground (Cox, 1961; see also Graham, 1961; Sakai et al., 1998; Verrier and Rochette, 2002; Jones and Maki, 2005). Therefore, the direction of magnetization is not restricted by the ambient geomagnetic field but by the magnetic field accompanying the lightning electric current in the ground.

Although the pattern of the electric current in the ground is not known very well, it has been suggested that the current flows for some distance in the horizontal direction and probably only for a short distance in the vertical direction (Sakai et al., 1998; Jones and Maki, 2005). If the time constant of the lightning current pulse is on the order of $1 \mu \mathrm{s}$ and the ground conductivity is $10^{-2} \mathrm{~S} / \mathrm{m}$, the depth of the penetration of the current into the ground can be roughly estimated to be at most $10 \mathrm{~m}$. A schematic picture of the current path and polarity of accompanying magnetic field is shown in Fig. 9.

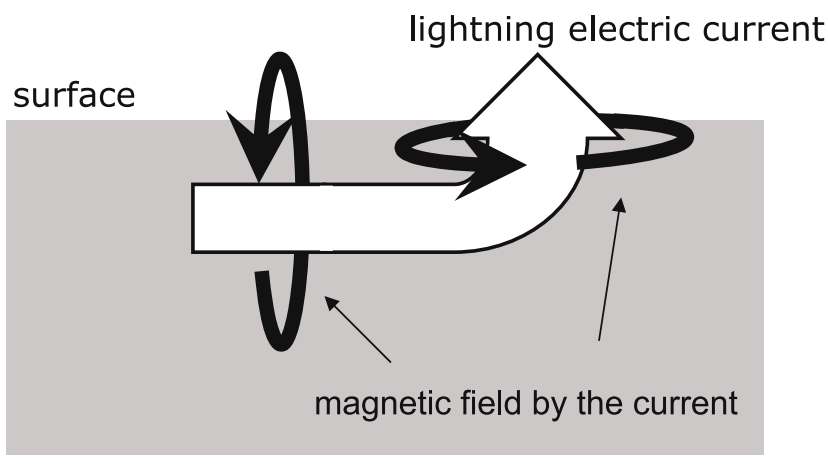

Fig. 9. Schematic picture of the path of lightning electric current (see Jones and Maki, 2005).

a)

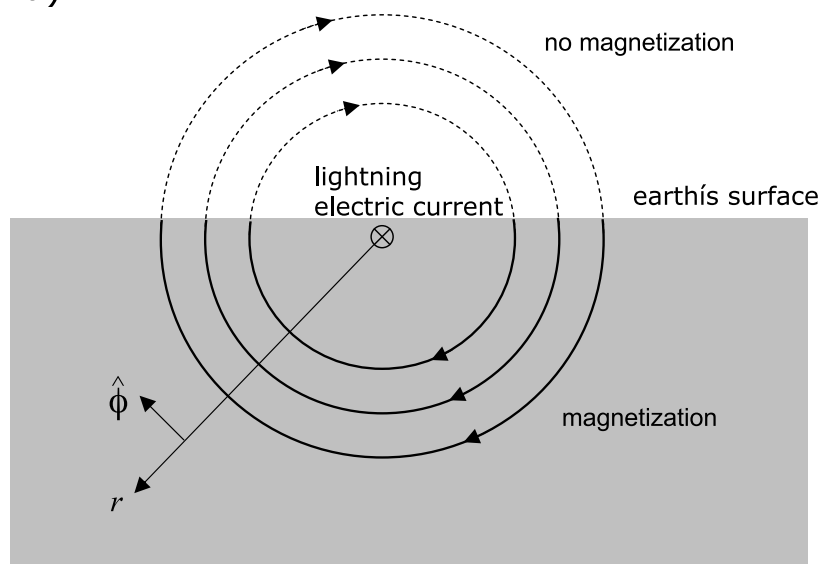

b)

horizontal lightning electric current

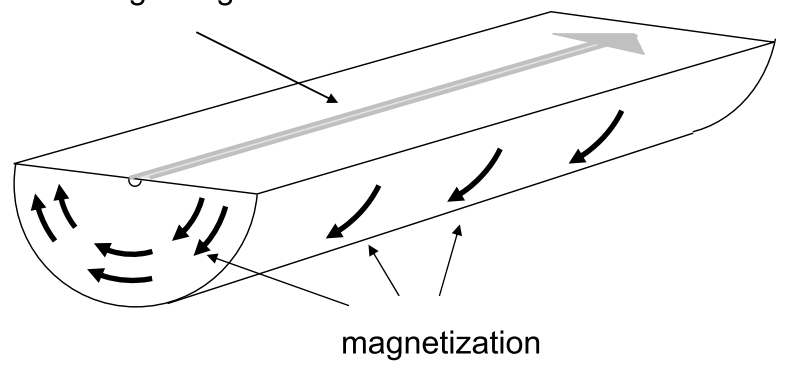

Fig. 10. (a) Direction of the magnetic field and magnetization due to horizontal lightning electric current. Only the part in the ground (shaded area) is magnetized by the current. (b) A model of magnetized body due to LIRM. Lightning electric current is assumed to flow along the axis of the cylinder which is right below the earth's surface.

It is likely that magnetization due to the vertical portion of the electric current can be modeled by continuous circular magnetization in the zonal direction (Fig. 9; also see Sakei et al. (1998) for distribution of observed magnetization at an archaeological ruin). If the magnetization depends only on the distance from the electric current and is uniform in the zonal direction, it does not produce a magnetic field outside of the region because $\nabla_{\mathbf{x}^{\prime}} \cdot \mathbf{J}\left(\mathbf{x}^{\prime}\right)=0$ everywhere in this case (see Eq. (3)). The heterogeneity of magnetization in the zonal direction is necessary to have a magnetic field outside of the magnetized region, and the ef- 
Table 2. Parameters used in the calculations. $B_{i}, B_{o}$ and $r_{i}$ are given, and $r_{o}$ is calculated from these and Eq. (6).

\begin{tabular}{c|c|l|c|c}
$B_{i}(\mathrm{mT})$ & $B_{o}(\mathrm{mT})$ & $r_{i} \mathrm{~m}$ & $r_{o} \mathrm{~m}(n=1)$ & $r_{o} \mathrm{~m}(n=2)$ \\
\hline 100 & 3 & 0.05 & 1.67 & 0.28 \\
100 & 3 & 0.1 & 3.33 & 0.58 \\
100 & 3 & 0.2 & 6.67 & 1.15 \\
100 & 1 & 0.1 & 10 & 1 \\
100 & 10 & 0.1 & 1 & 0.32
\end{tabular}

Table 3. Magnetization necessary to produce peak-to-peak amplitude of $80 \mathrm{nT}$.

\begin{tabular}{c|c|c} 
Cases & $J_{o} \mathrm{~A} / \mathrm{m}(n=1)$ & $J_{o} \mathrm{~A} / \mathrm{m}(n=2)$ \\
\hline$B_{i} / B_{o}=100 / 3 r_{i}=0.05 \mathrm{~m}$ & 35.6 & 167.0 \\
$B_{i} / B_{o}=100 / 3 r_{i}=0.1 \mathrm{~m}$ & 17.4 & 46.9 \\
$B_{i} / B_{o}=100 / 3 r_{i}=0.2 \mathrm{~m}$ & 11.5 & 15.4 \\
$B_{i} / B_{o}=100 / 1 r_{i}=0.1 \mathrm{~m}$ & 31.1 & 40.8 \\
$B_{i} / B_{o}=100 / 10 r_{i}=0.1 \mathrm{~m}$ & 22.4 & 61.2
\end{tabular}

fective magnetization may be modeled by several horizontal dipoles. However, this magnetization model does not seem to be suitable for producing a coherent magnetic field structure, such as the anomalous patch-pair in YMO.

Although the horizontal electric current generates a magnetic field in the region above and below the surface of the earth, only that part below the surface can receive magnetization due to the field (see Fig. 10(a)). A distribution of LIRM similar to that in Fig. 10(a) was actually observed by Graham (1961). The divergence of the magnetization vector in this case is not zero at the surface of the earth; therefore, the magnetic field should appear outside of the magnetized region according to Eq. (3). If the horizontal electric current flows at the surface of the earth for some distance, the effective magnetized region should have a form of a semicylinder, as shown in Fig. 10(b).

Let us now model the magnetic field anomaly generated by LIRM. Suppose that the lightning electric current flowed horizontally at the surface of the earth. In this case, the magnetized region can be represented by a semi-cylindrical body, as shown in Fig. 10(b). We also assume that the strength of the magnetic field due to the lightning current is much stronger than that of the ambient magnetic field and that the region close to the electric current is completely remagnetized by LIRM. Because the intensity of the magnetization is a function of the strength of the magnetic field due to the lightning current, the magnetization vector in the semi-cylindrical body may be written as

$$
\mathbf{J}_{M}=J\left(B_{L}(r)\right) \hat{\phi}
$$

in the ground, where $r$ is the distance from the axis of the semi-cylinder (from the horizontal lightning electric current), $B_{L}(r)$ is the intensity of magnetic field due to the lightning current at $r$ and $\hat{\phi}$ is a unit vector in the zonal direction (see Fig. 10(a)).

If $B_{L}(r)$ is larger than a certain critical value $\left(=B_{i}\right)$, the magnetization obtained at the position is that of saturation isothermal remanent magnetization (SIRM). Also, if $B_{L}(r)$ is smaller than another critical value $\left(=B_{o}\right)$, the IRM due to the field is zero. By considering these relationships, we model the magnetization as the following;

$$
J(r)=\left\{\begin{array}{lll}
0 & \text { if } & r_{o}<r \\
J_{0} \frac{B_{L}(r)-B_{o}}{B_{i}-B_{o}} & \text { if } & r_{i} \leq r \leq r_{o} \\
J_{0} & \text { if } r<r_{i}
\end{array}\right.
$$

where $r_{i}$ and $r_{o}$ are the radii for which $B_{L}$ is equal to $B_{i}$ and $B_{o}$, respectively, and $J_{0}$ is the SIRM intensity. Although $B_{L}(r)$ may have a complicated form, we assume a simple form as

$$
B_{L}(r)=B_{i}\left(\frac{r_{i}}{r}\right)^{n}, \quad n=1 \text { or } 2 .
$$

The strength of the magnetic field generated by a straight electric current behaves as $r^{-1}\left(r^{-2}\right)$ if the length of the originating electric current is infinitely long (short). The form in Eq. (6) with $n=1$ or 2 represents the end members of the magnetic field and magnetization distributions. Such dependencies on $n$ are found in actual LIRM (Sakai et al., 1998).

We are going to assume values of $n, r_{i}, B_{i}, B_{o}$ and $J_{0}$ to calculate the magnetic field and magnetic anomalies in the following section. $r_{o}$ is automatically determined by setting $B_{i}, B_{o}$ and $n$ (see Eq. (6)). Note that it is only necessary to specify the ratio $B_{i} / B_{o}$, rather than both $B_{i}$ and $B_{o}$, to determine the magnetization profile in Eq. (5).

\section{Results and Discussion}

We first calculated the magnetic field generated by the magnetization represented by Eqs. (4), (5) and (6) and then obtained the magnetic intensity anomaly at a height of $2 \mathrm{~m}$ in the YMO condition ( $H=29900 \mathrm{nT}, Z=35400 \mathrm{nT})$, i.e. in reference to the intensity of $46337.6 \mathrm{nT}\left(=\sqrt{H^{2}+Z^{2}}\right)$. The direction of the lightning electric current is assumed to be from east to west, i.e. the semi-cylindrical body lies in 


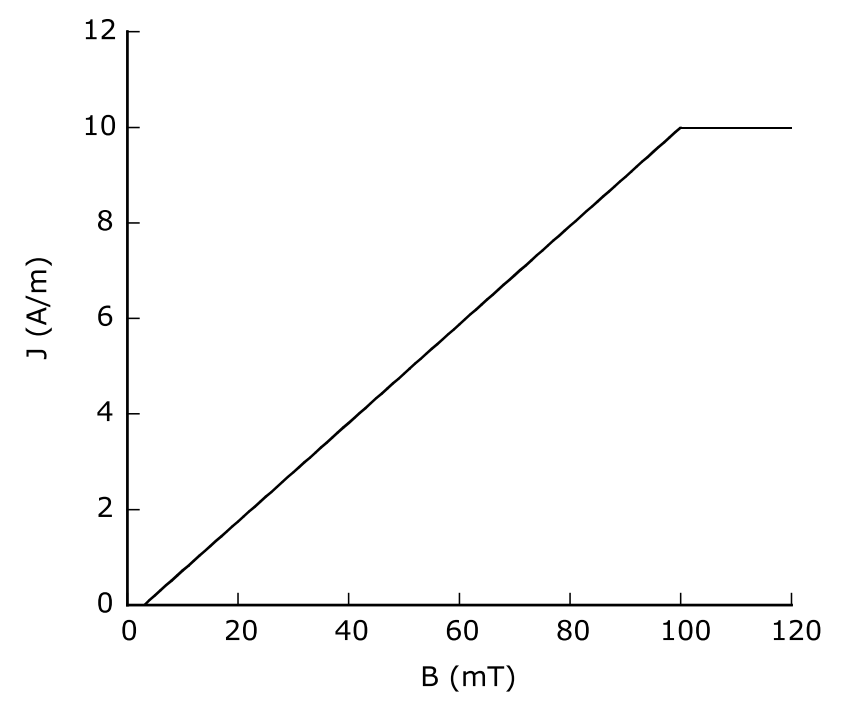

Fig. 11. Relationship between intensity of magnetization $J$ and magnetic field $B$ when $J_{0}=10 \mathrm{~A} / \mathrm{m}, B_{i}=100 \mathrm{mT}$ and $B_{o}=3 \mathrm{mT}$; see Eq. (5).

the east-west direction, and it is assumed that the length of the magnetized body in the direction is $30 \mathrm{~m} . J_{0}=10 \mathrm{~A} / \mathrm{m}$, and the values in Table 2 are assumed for the calculations (see Wasilewski and Kletetschka, 1999). The relationship between $B_{L}$ and $J$ (Eq. (5)) is shown in Fig. 11 for the case with $J_{0}=10 \mathrm{~A} / \mathrm{m}, B_{i}=100 \mathrm{mT}$ and $B_{o}=3 \mathrm{mT}$.

Figure 12 shows the calculated magnetic anomaly for $n=1$ (Fig. 12(a)) and $n=2$ (Eq. 12(b)) with condition $B_{i}=100 \mathrm{mT}, B_{o}=3 \mathrm{mT}$, and $r_{i}=0.1 \mathrm{~m}$. The thin character of the anomalous patch-pair at the site is well reproduced by the semi-cylindrical magnetization model with $n=1$ and 2 . The zero line of the magnetic anomaly is shifted southward from the location of supposed lightning electric current because of the ambient magnetic field. The amplitude of anomaly is smaller when $n=2$ than when $n=1$ because the magnetization of the case with $n=2$ decays faster than the case with $n=1$ with increasing $r$. The amplitude of the peak depends almost linearly on $J_{0}$ in this regime (magnetic field due to magnetized body $\ll$ ambient magnetic field) so that we can estimate that the peak-to-peak amplitude of the observed patch-pair, about $80 \mathrm{nT}$, can be reproduced with $J_{0}=17 \mathrm{~A} / \mathrm{m}$ for $n=1$ and $47 \mathrm{~A} / \mathrm{m}$ for $n=2$ (see Table 3 for $J_{0}$ for the other cases).

Figure 13(a) and (b) shows the calculated profiles of magnetic anomaly crossing the patch-pair (along $y=0$ in Fig. 12(a) and (b)) for $n=1$ and $n=2$, respectively. Profiles corresponding to all cases listed in Table 2 are plotted to examine parameter dependencies of the profile. The broadening of the structure is seen if $n=1$, especially with $r_{i}=0.2 \mathrm{~m}$ or $B_{o}=1 \mathrm{mT}$. Both cases correspond to large $r_{o}$ where the calculated patch-pairs are not contained in a 15 $\mathrm{m}$ range in the north-south direction. On the contrary, the distributions when $n=2$ do not change significantly (except the amplitude) because the magnetization is confined to within a distance of about $1 \mathrm{~m}$ for all the cases (see $r_{o}$ $(n=2)$ in Table 2) due to the fast decay of the source magnetic field with $r$. The separation of the peaks of patch-pair is about $5 \mathrm{~m}$, and the patch-pair is confined to within $15 \mathrm{~m}$ for cases with $n=2$.
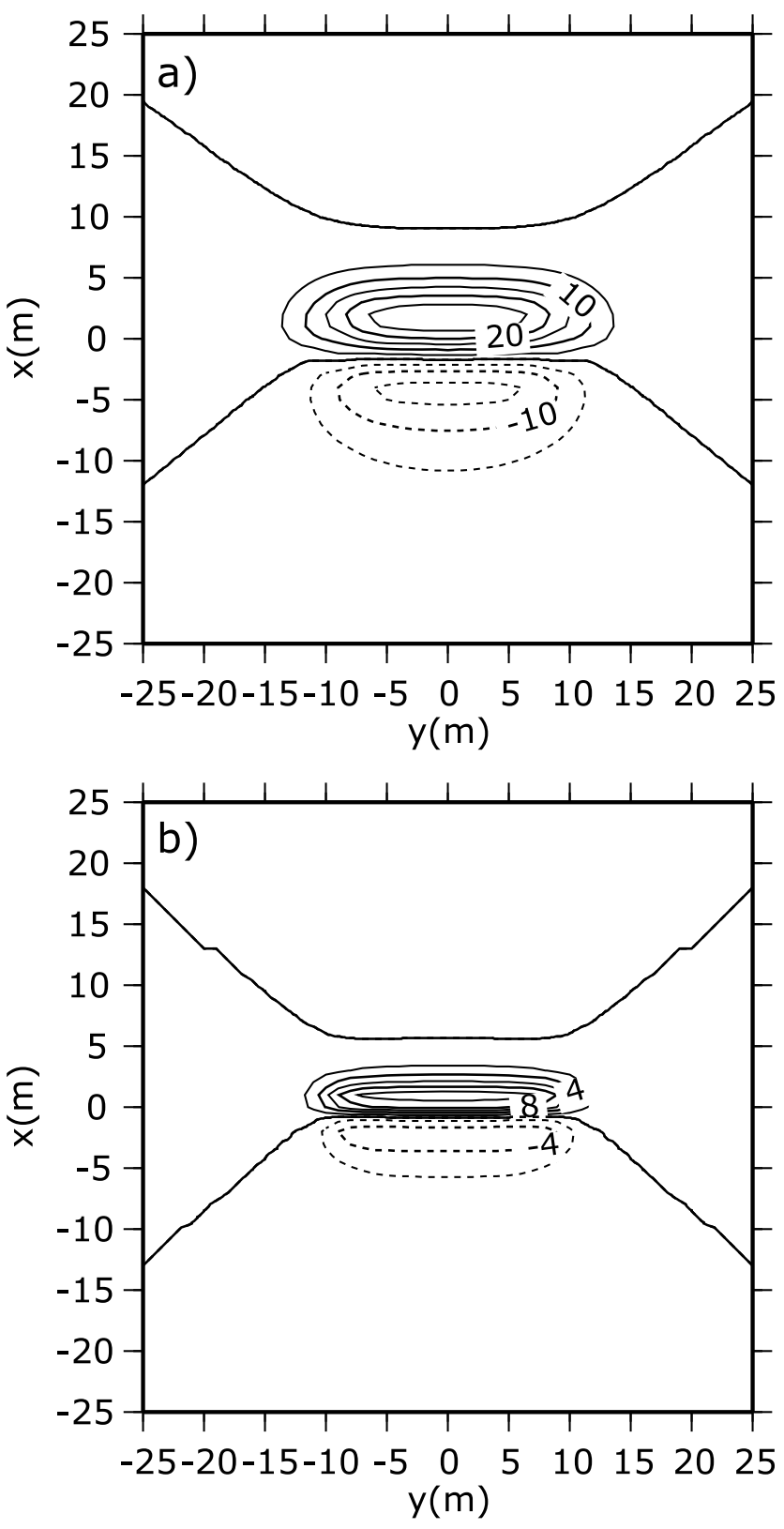

Fig. 12. Distribution of magnetic anomaly generated by model magnetizaion (see Eqs. (4), (5), (6) and Fig. 10(b)). North is in positive $x$ direction. The lightning electric current is assumed to flow from east to west, and the axis of the semi-cylindrical magnetized body corresponds to $x=0$. The length of the magnetized body (semi-cylinder) is assumed to be $30 \mathrm{~m}$ occupying $-15 \mathrm{~m}<y<15 \mathrm{~m}$ region. The ambient field is supposed the values at YMO $(H=29,900 \mathrm{nT}, Z=35,400 \mathrm{nT})$. $J_{0}=10 \mathrm{~A} / \mathrm{m}, B_{i}=100 \mathrm{mT}, B_{o}=3 \mathrm{mT}$ and $r_{i}=0.1 \mathrm{~m}$ are assumed. (a) $n=1$ case, (b) $n=2$ case. Unit: $\mathrm{nT}$.

Figure 14 compares the observed (Line B in Fig. 3) and calculated profiles of magnetic anomaly crossing the patchpair. $B_{i}=100 \mathrm{mT}, B_{o}=3 \mathrm{mT}$ and $r_{i}=0.1 \mathrm{~m}$ are assumed, and the $J_{0}$ 's are adjusted so that the calculated profiles have roughly the same peak-to-peak amplitude with the observed profile $(17 \mathrm{~A} / \mathrm{m}$ for $n=1$ and $47 \mathrm{~A} / \mathrm{m}$ for $n=2)$. The observed distribution of the patch-pair fits well with the case with $n=1$. It is most likely that the observed patchpair is due to LIRM with $n=1$. Although it is beyond the scope of the present investigation, palaeomagnetic/rock magnetic laboratory testing (see Maki, 2005; Verrier and 

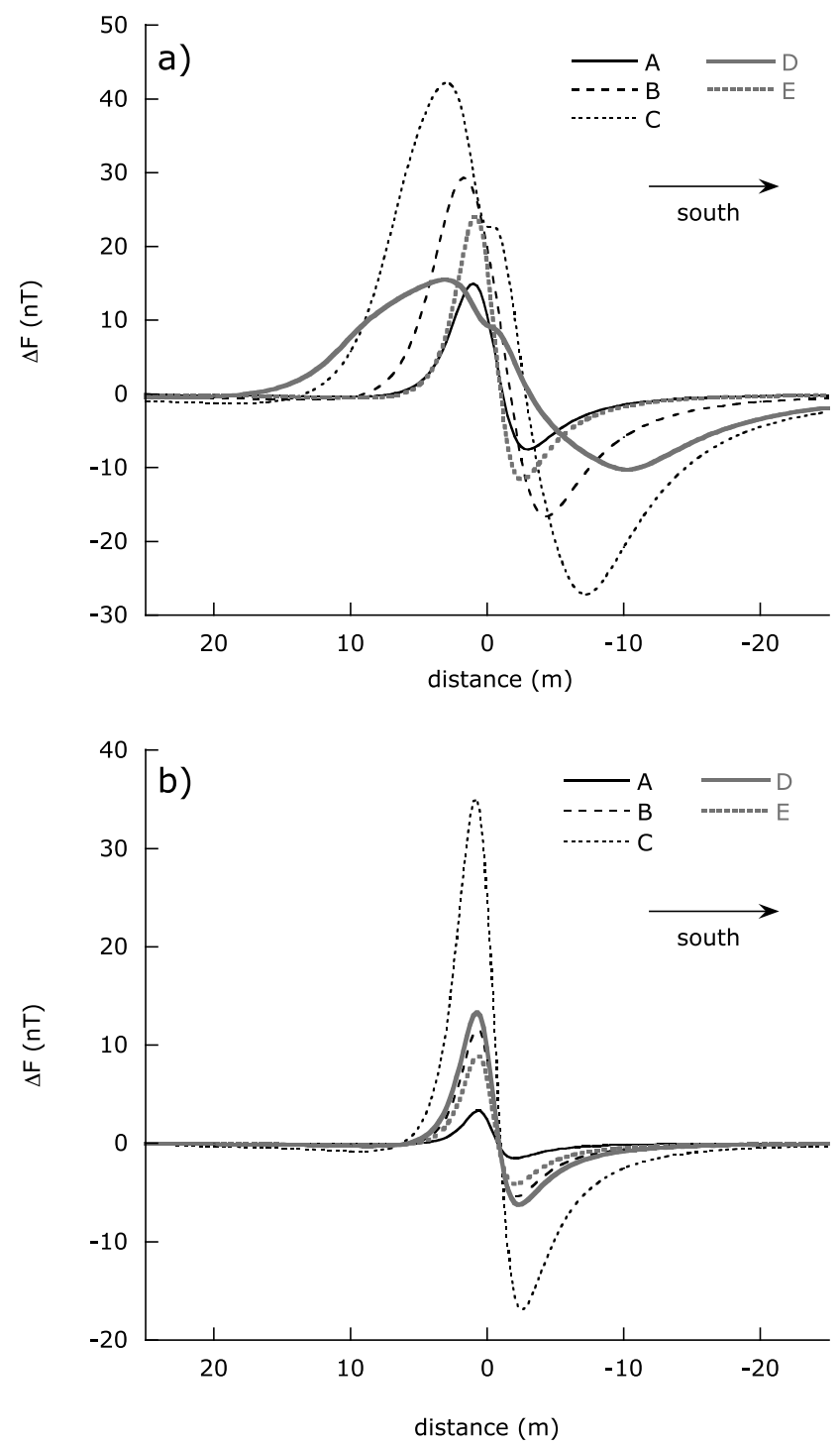

Fig. 13. Calculated profiles of magnetic field anomaly along $y=0$ (see Fig. 12). (a) $n=1$ and (b) $n=2 . J_{0}=10 \mathrm{~A} / \mathrm{m}$ is assumed for all cases. The other parameters are set at the following. A: $B_{i}=100 \mathrm{mT}$, $B_{o}=3 \mathrm{mT}, r_{i}=0.05 \mathrm{~m} ; \mathrm{B}: B_{i}=100 \mathrm{mT}, B_{o}=3 \mathrm{mT}, r_{i}=0.1 \mathrm{~m} ; \mathrm{C}:$ $B_{i}=100 \mathrm{mT}, B_{o}=3 \mathrm{mT}, r_{i}=0.2 \mathrm{~m} ; \mathrm{D}: B_{i}=100 \mathrm{mT}, B_{o}=1 \mathrm{mT}$, $r_{i}=0.1 \mathrm{~m} ; \mathrm{E}: B_{i}=100 \mathrm{mT}, B_{o}=10 \mathrm{mT}, r_{i}=0.1 \mathrm{~m}$.

Rochette, 2002) of soil samples taken from the magnetized area would confirm whether the spatial distribution of the magnetization is consistent with LIRM generated by a horizontal electric current.

Magnetic anomaly at a height of $1.5 \mathrm{~m}$ made by LIRM with $n=1, J_{0}=17 \mathrm{~A} / \mathrm{m}, B_{i}=100 \mathrm{mT}, B_{o}=3 \mathrm{mT}$ and $r_{i}=0.1 \mathrm{~m}$ is calculated and compared with that at a height of $2.0 \mathrm{~m}$ obtained previously in order to examine if the height difference of the observations would generate the observed difference shown in Fig. 7. The difference between the two calculated profiles (magnetic anomaly at a height of $2.0 \mathrm{~m}$ - that at $1.5 \mathrm{~m}$ ) shows the negative-positive patch-pair in the north-south direction as having a peakto-peak amplitude of about $20 \mathrm{nT}$. This is comparable to what is found in Fig. 7 near the anomalous patch, and the observed difference could be due to the height difference. However, as mentioned in Section 2, the effect of the loca-
Observed and calculated $\Delta F$ profiles

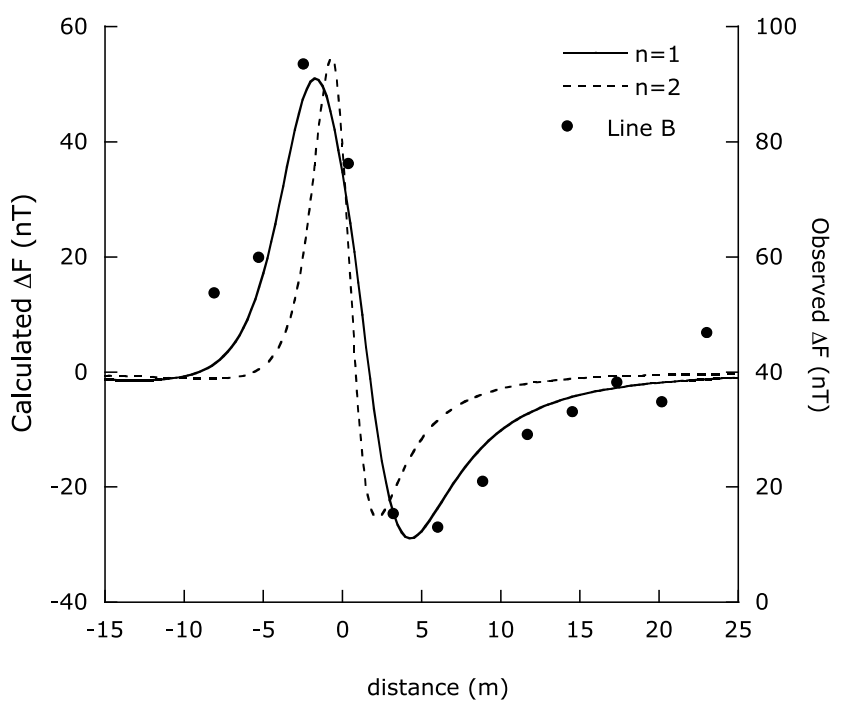

Fig. 14. Observed and calculated magnetic anomaly profiles crossing the patch-pair. Observed profile along Line B (black dots, Fig. 3) and calculated profiles with $J_{0}=10 \mathrm{~A} / \mathrm{m}, B_{i}=100 \mathrm{mT}, B_{o}=3 \mathrm{mT}$ and $r_{i}=0.1 \mathrm{~m}$ are shown. Peak-to-peak amplitudes of calculated profiles are adjusted by changing $J_{0}$ to that of observed profile. $J_{0}=17 \mathrm{~A} / \mathrm{m}$ if $n=1$ and $47 \mathrm{~A} / \mathrm{m}$ if $n=2$.

tion error cannot be disregarded in the present case. Location errors of several tens of centimeters for 2005 and that of $1 \mathrm{~m}$ for 1982 can easily produce an apparent error of about $20 \mathrm{nT}$ at grid points where the gradient is as large as 15 $\mathrm{nT} / \mathrm{m}$. In either case, it is not required to introduce a change of magnetization over time to explain the observed difference in Fig. 7. Together with small average difference, 3.7 $\mathrm{nT}$, between the two, we may conclude that there have been no significant changes in the magnetic environment since 1982. This indicates that the LIRM in the observatory area has been stable against external causes such as freeze/thaw cycles and/or bioturbation since the time.

\section{Conclusions}

The magnetic anomaly (intensity) maps reveal that an anomalous patch-pair occurs in YMO sometime between 1975 and 1982 and the patch-pair has existed stably since then. The peak-to-peak amplitude of the patch-pair is about $80 \mathrm{nT}$ if observed at a height of $2 \mathrm{~m}$. The patch-pair is confined to $15 \mathrm{~m}$ in the direction crossing the patches, but the lateral extent is longer, about $30 \mathrm{~m}$. The observed difference between the magnetic anomaly maps of 1982 and 2005 is due to the difference in the observation height of magnetizations near the surface and/or the location errors made during the two sets of measurements. The magnetic environment in the observatory area has been stable since 1982.

The patch-pair is most likely produced by the LIRM acquired by the lightning strike on July 16, 1981. The maximum magnetization required to explain the peak-to-peak amplitude of the patch-pair is estimated to be approximately $20 \mathrm{~A} / \mathrm{m}$ for the best-fit model with $n=1, B_{i}=100 \mathrm{mT}$, $B_{o}=3 \mathrm{mT}$ and $r_{i}=0.1 \mathrm{~m}$, which is equivalent to a semicylindrical magnetization of about $6 \mathrm{~m}$ in diameter. The 
magnetization will be in the range of $10-40 \mathrm{~A} / \mathrm{m}$ if $n=1$ (long electric current) is assumed. Model magnetization with $n=2$ (short electric current) cannot reproduce the observed feature of the patch-pair well.

Acknowledgments. We would like to thank two reviewers, Drs. David Maki and Pierre Rochette, for their constructive comments to improve the paper.

\section{References}

Aida, N., Natural remanent magnetizations and ferromagnetic minerals of the late pleistocene volcanic ash ("younger loam") of the Yatsugatake volcanoes, central Japan, Quat. Res., 17, 86-94, 1978 (in Japanese with English abstract).

Cox, A., Anomalous remanent magnetization of basalt, U.S. Geol. Surv. Bull., 1083-E, 131-160, 1961.

Graham, K. W. T., The re-magnetization of a surface outcrop by lightning currents, Geophys. J. Roy. Astron. Soc., 6, 85-102, 1961.

Jones, G. and D. L. Maki, Lightning induced magnetic anomalies on archaeological sites, Archaeol. Prospect., 12, 191-197, 2005.
Maki, D., Lightning strikes and prehistoric ovens: Determining the source of magnetic anomalies using techniques of environmental magnetism, Geoarcheology, 20, 449-459, 2005.

Sakai, H., S. Sunada, and H. Sakurano, Study of lightning current by remanent magnetization, Elec. Eng. in Japan, 123, 1050-1055, 1997.

Utada, H. and S. Koyama, Variation of magnetic intensity at Yatsugatake geomagnetic observatory due to lightning strike (July, 1981), Proceedings of Conductivity Anomaly Research, 181-186, 1982. (in Japanese)

Verrier, V. and P. Rochette, Estimating peak currents at ground lightning impacts using remanent magnetzation, Geophys. Res. Lett., 29(18), 1867, doi:10.1029/2002GL015207, 2002.

Wasilewski, P. and G. Kletetschka, Lodestone: Natures only permanent magnet-What it is and how it gets charged, Geophys. Res. Lett., 26(15), 2275-2278, 1999.

Yukutake, T., S. Koyama, and T. Yoshino, Geomagnetic environment of Yatsugatake geomagnetic observatory, Bull. Earthq. Res. Inst., 50, 7381, 1975 (in Japanese with English abstract).

H. Shimizu (e-mail: shimizu@eri.u-tokyo.ac.jp), T. Koyama, S. Koyama, and H. Utada 